\title{
Efficacy of gel-based artificial saliva on Candida colonization and saliva properties in xerostomic post-radiotherapy head and neck cancer patients: a randomized controlled trial
}

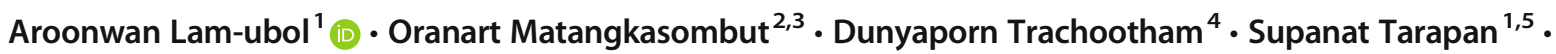 \\ Vanthana Sattabanasuk ${ }^{6}$. Sineepat Talungchit ${ }^{1}$. Wannaporn Paemuang ${ }^{7} \cdot$ Tawaree Phonyiam $^{4}$. \\ Orapin Chokchaitam ${ }^{8} \cdot$ On-ong Mungkung ${ }^{8}$
}

Received: 15 June 2020 / Accepted: 29 July 2020 / Published online: 10 August 2020

(C) The Author(s) 2020

\begin{abstract}
Objective To evaluate the efficacy of an edible artificial saliva gel, oral moisturizing jelly (OMJ), and a topical commercial gel (GC dry mouth gel) on Candida colonization and saliva properties.

Materials and methods This study was a secondary analysis of a single-blinded randomized controlled trial conducted in xerostomic post-radiotherapy head and neck cancer patients. Candida colonization, stimulated salivary flow rate (SSFR), saliva $\mathrm{pH}$, and buffering capacity (BC) were measured at 0,1 , and 2 months after each intervention. Candida colonization was quantified by colony counts and species identified by Candida Chromagar, polymerase chain reaction, and API 20C AUX system. Statistical significance level was 0.05 .

Results A total of 56 participants in $\mathrm{OMJ}(N=30)$ and $\mathrm{GC}(N=26)$ groups completed the study. OMJ significantly increased saliva $\mathrm{pH}(p=0.042)$ and BC $(p=0.013)$ after 1 -month use, while GC only improved saliva $\mathrm{pH}(p=0.027)$. Both interventions tended to increase SSFR but only GC had a significant increase at 2 months $(p=0.015)$. GC and OMJ significantly decreased the number of Candida species at 1 and 2 months, respectively. Both groups tended to reduce Candida counts but not significant. Conclusions Both OMJ and GC saliva gels could improve saliva $\mathrm{pH}$ and decrease the number of Candida species. OMJ is superior to GC in its buffering capacity, while GC may better improve salivary flow rate. Long-term and large-scale study is warranted to test the efficacy of artificial saliva in oral health improvement.

Clinical relevance OMJ and GC gel could decrease the number of Candida species and improve saliva properties in postradiation xerostomic patients.
\end{abstract}

Trial registration number Clinicaltrials.gov NCT03035825. Date of registration: 25th January 2017.

Keywords Xerostomia $\cdot$ Artificial saliva $\cdot$ Head and neck cancer $\cdot$ Radiotherapy $\cdot$ Candida $\cdot$ Buffering capacity

\section{Introduction}

Radiotherapy for head and neck cancer commonly causes destruction and fibrosis of salivary glands leading to

Aroonwan Lam-ubol

aroonwan@gmail.com

1 Department of Oral Surgery and Oral Medicine, Faculty of Dentistry, Srinakharinwirot University, 114 Sukhumvit 23, Wattana, Bangkok 10110, Thailand

2 Department of Microbiology and Research Unit on Oral Microbiology and Immunology, Faculty of Dentistry, Chulalongkorn University, Bangkok, Thailand hyposalivation $[1,2]$. The quantity and quality of saliva in head and neck cancer patients are dramatically declined after completion of radiotherapy. Their salivary flow rate, $\mathrm{pH}$, and buffering capacity are abnormally low [3, 4]. Consequently,

3 Laboratory of Biotechnology, Chulabhorn Research Institute, Bangkok, Thailand

4 Institute of Nutrition, Mahidol University, Nakhon Pathom, Thailand

5 Langsuan Hospital, Chumphon, Thailand

6 Faculty of Dentistry, Mahidol University, Bangkok, Thailand

7 Department of Vascular Surgery, Faculty of Medicine Siriraj Hospital, Mahidol University, Bangkok, Thailand

8 Chonburi Cancer Hospital, Chonburi, Thailand 
the patients usually suffer from dry mouth symptoms (xerostomia) as well as poor oral conditions $[2,5,6]$.

Among important oral health problems, such as dental caries, atrophic mucosa, altered taste sensation, and traumatic ulcer, candidiasis is one of the most common oral sequelae in post-radiotherapy head and neck cancer patients [5-7]. Even without any symptoms or clinical diagnosis of Candida infection, xerostomic post-radiotherapy head and neck cancer patients were reported to have increased Candida colonization $[4,8]$. The amplified colonization and poor saliva properties together with immunocompromised host pose head and neck cancer patients at high risk of candidiasis $[9,10]$. Candida albicans is the most common oral species detected in both healthy and xerostomic individuals [11-14]. However, xerostomic patients are also colonized by non-albicans species or multiple Candida species, leading to a more complex oral environment and treatment difficulty $[7$, $15,16]$.

Current management strategies for hyposalivation (reduced salivary flow) include systemic and topical options. However, systemic therapies have side effects and are ineffective for patients with impaired salivary tissue. Topical treatments, including the use of artificial saliva, are therefore more commonly recommended $[1,17,18]$. Several artificial saliva products are commercially available [19]. To our knowledge, all of them are for oral lubrication and not recommended to be swallowed. Previous studies showed that various types of artificial saliva can relieve signs and symptoms of dry mouth to some extent [20-24]. However, most of those studies were not randomized and were short-term (2-4 weeks) [20-24]. Moreover, the effects of artificial saliva on Candida colonization are unclear.

A novel artificial saliva called oral moisturizing jelly (OMJ) was developed by the Dental Innovation Foundation under Royal Patronage, a non-profit organization in Thailand. The product used food-grade ingredients without any preservative agents. Therefore, it can be swallowed and provides lubrication from the oral cavity through the throat, similarly to natural saliva. Our previous pre-post study evaluated the efficacy of OMJ in xerostomic elderly patients with systemic diseases. The results demonstrated that continuous intake of OMJ for 1-2 months significantly reduced signs and symptoms of dry mouth, and achieved more than $80 \%$ satisfaction [25]. In addition, continuous use of OMJ for 1 month prevented the decline of $\mathrm{pH}$ and improved buffering capacity [25]. However, the effects of OMJ on Candida colonization and saliva properties of xerostomic post-radiotherapy head and neck cancer patients are unknown.

This report was a secondary analysis of a single-blinded randomized controlled trial in xerostomic post-radiotherapy head and neck cancer patients [26]. We previously published that continuous use of OMJ for a month improved signs and symptoms of dry mouth, and increased swallowing ability
[26]. In this study, we aimed to analyze the effects of OMJ, in comparison with a commercially available gel-based artificial saliva (GC dry mouth gel), on salivary flow rates, saliva $\mathrm{pH}$, and buffering capacity as well as Candida colonization in xerostomic post-radiotherapy head and neck cancer patients.

\section{Methods}

This was a secondary analysis of a single-blinded randomized controlled trial conducted as previously described [26]. The study was performed according to the Declaration of Helsinki and ICH-GCP. The Ethical committee of Chonburi Cancer Hospital (IRB number 7/2559); Faculty of Dentistry, Srinakharinwirot University (DENTSWU-EC26/2560); and Institute of Nutrition, Mahidol University (MU-CIRB 2017/165.0811), approved the study protocol. All participants signed written informed consent prior to data collection. The study protocol was registered at Clinical trial.gov (clinicaltrials.gov number NCT03035825).

\section{Study population}

The trial included 72 post-radiotherapy head and neck cancer patients with xerostomic problems ( $N=37$ for OMJ and 35 for GC (control) groups) [26]. However, in this study, we analyzed data from a subpopulation of 56 who could provide saliva samples at all time points. The participants were recruited from three sites including Chonburi Cancer Hospital; Faculty of Dentistry, Srinakharinwirot University; and Institute of Nutrition, Mahidol University, Thailand. Inclusion criteria included patients who had a history of head and neck cancer, are 30-70 years old, had finished radiotherapy for at least 1 month and/or chemotherapy for at least 2 weeks, are able to use/consume the interventions without choking, can communicate in Thai, and had subjective dry mouth score $\geq 3$ according to the questionnaire used in our previous study $[25,26]$. Exclusion criteria were patients with mucositis grade $\geq 1$, clinically diagnosed candidiasis, or systemic diseases associated with hyposalivation, such as Sjögren's syndrome, or those taking drugs with anticholinergic effects such as pilocarpine and anti-depressants. Moreover, participants with normal saliva $\mathrm{pH}$ and high buffering capacity at baseline were excluded from the analysis. In addition, participants who did not come for both follow-up visits or developed cancer recurrence or allergy to the interventions were discontinued from the study.

\section{Study design, interventions, blinding, and randomization}

The protocol of the trial was described in Nuchit et al. [26]. Briefly, the participants were randomly allocated to OMJ or 
commercially available GC dry mouth gel using a minimization method to match age, sex, and baseline subjective dry mouth score between groups. OMJ is manufactured by Dental Innovation Foundation under Royal Patronage, Thailand. While GC dry mouth gel is a product of GC Corp., Japan, groups. All examiners involved in data collection and statistical analysis were blinded. Participants were instructed to take 1-2 teaspoons of OMJ, hold in the mouth for a few seconds, and swallow. Participants in the GC group were instructed to apply approximately $500 \mathrm{mg}$ (pea-sized drop) of GC dry mouth gel as a thin layer throughout the oral cavity. Both groups were asked to use the products 6 times per day or every $3 \mathrm{~h}$ for 2 months.

\section{Sample size and power calculation}

The sample size of this study was calculated as described [26]. To ensure that this secondary data analysis had adequate power, post hoc power analyses for saliva properties and Candida colonization were performed using $\mathrm{G}$ power. A power of 0.87 , 0.9 , and 0.92 was obtained for the data of saliva $\mathrm{pH}$, buffering capacity, and the number of Candida species, respectively.

\section{Data collection}

The outcome measures, including salivary flow rates, saliva pH and buffering capacity, Candida counts, and Candida species, were evaluated at 0,1 , and 2 months after interventions. Demographic data, including medical history, cancer sites, detail of cancer treatment, and history of antibiotic and antifungal drug use, were retrieved from treatment records and by interviewing the participants. Subjective dry mouth scores were obtained by using a validated questionnaire as described $[25,26]$.

\section{Salivary flow rates, $\mathrm{pH}$, and buffering capacity}

Stimulated saliva was collected as described previously [4, 25]. Participants were asked to chew on a piece of paraffin for $1 \mathrm{~min}$. Whole stimulated saliva was collected for $10 \mathrm{~min}$ into a sterile $50-\mathrm{ml}$ tube. Stimulated salivary flow rates were calculated as milliliters per minute. Then, the $\mathrm{pH}$ and buffering capacity were measured by using Seven $2 \mathrm{Go} \mathrm{pH}$ meter $\mathrm{S} 2$ (Mettler Toledo, Switzerland) equipped with ultramicroelectrode. A saliva $\mathrm{pH}$ of less than 6.8 was considered acidic [25]. Buffering capacity was measured using Ericsson's method as previously described with modification [27]. Briefly, $50 \mu \mathrm{l}$ of saliva was mixed with $150 \mu \mathrm{l}$ of $5-\mathrm{mM}$ $\mathrm{HCl}$. After 10-min incubation, $\mathrm{pH}$ of the mixture was measured by the $\mathrm{pH}$ meter. Final $\mathrm{pH}$ of $\leq 4,4.1-5.5$, and $\geq 5.6$ were considered low, moderate, and high buffering capacity, respectively [27].

\section{Candida counts and species identification}

Candida colonization was evaluated from oral rinse samples. Participants were asked to orally gargle with $10 \mathrm{ml}$ of $0.01-\mathrm{M}$ sterile phosphate-buffered saline, $\mathrm{pH} 7.2$ for $5 \mathrm{~min}$ as described previously [4]. After collection of the oral rinse, the samples were placed on ice and transported to the laboratory for analysis within $6 \mathrm{~h}$. After centrifugation at $3000 \mathrm{rpm}$ for $7 \mathrm{~min}$, undiluted and 1:10 diluted samples were cultured at $37^{\circ} \mathrm{C}$ for $48 \mathrm{~h}$ on Sabouraud dextrose agar (Himedia, Mumbai, India) and CHROMagar Candida (Chromagar company, Paris, France), respectively. The number of colonyforming units (CFUs) and colony color and morphology were recorded. Plates exhibiting no growth were incubated for an additional $24 \mathrm{~h}$ to confirm the absence of Candida colonies.

Candida species were initially evaluated by color as appeared on CHROMagar Candida (C. albicans, green; Candida tropicalis, metallic blue; Candida krusei, pink, fuzzy; other species, white to mauve). The colonies were then isolated for polymerase chain reaction (PCR) to identify the following species using species-specific primers as specified in the parentheses according to previous studies $[4,14,28$, 29]: C. albicans (CAL5-NL4CAL), Candida glabrata (CGL1-NL4CGL1), Candida parapsilosis (CTA4NL4LEL1), and Candida dubliniensis (CDU2-NL4CAL). The remaining species were identified by the API 20C AUX yeast identification system (bioMérieux, Marcy L'Etoile, France).

\section{Statistical analysis}

Comparisons of baseline data between OMJ (study) and GC (control) groups were evaluated by Chi-square test or Fisher's exact test for categorical data, and independent $t$ test or MannWhitney $U$ test for continuous data, as specified. Comparisons of numerical outcome measures at baseline and first and second follow-up visits of the same group were analyzed by repeated measure ANOVA or related samples Friedman's test with Bonferroni correction for multiple comparisons. Comparisons of categorical outcome measures among baseline and first and second follow-up visits of the same group were analyzed by chi-square test or Fisher's exact test. The prevalence of Candida species was analyzed using descriptive statistics. Correlation between $\log \mathrm{CFU}$ and saliva properties was analyzed by Pearson correlation analysis. All analyses were performed with the IBM SPSS statistics version 22 and GraphPad Prism version 8. A $p$ value of less than 0.05 was considered statistically significant. Post hoc power analysis was performed by the G Power version 3.1.9.3. The normality of data distributions was analyzed by the Shapiro-Wilk test. Parametric statistical tests were used only when the data passed normality test $(p>0.05)$. 


\section{Results}

\section{Characteristics of study population}

As shown in Fig. 1, a total of 72 participants ( $N=37$ for OMJ and 35 for GC) were initially recruited and randomized in the trial as described [26]. Sixteen participants dropped out during the trial due to unavailability on appointment date and transportation difficulties ( 6 and 8 participants in the OMJ and GC groups, respectively) and cancer recurrence (1 participant in each group). Finally, 30 participants in the OMJ group and 26 participants in the GC group provided saliva samples at all time points and were included in this study. All participants received definitive radiotherapy for head and neck cancer, mainly by conventional (2-dimensional) technique $(66 \%)$. The most common cancer sites were nasopharynx (35.7\%), followed by oral cavity $(28.6 \%)$ and tongue (17.9\%). The majority of participants was male (67.9\%) and had finished radiotherapy more than 1 year prior to enrollment (66.1\%). Twelve participants (21.4\%) had a history of antibiotics use within 1 month before recruitment, and 6 participants $(10.7 \%)$ had a history of antifungal use within 1 year before recruitment. Twelve participants $(21.4 \%)$ wore removable dental prostheses. As shown in Table 1, baseline characteristics, including dry mouth symptoms, saliva quantity and quality, and Candida status, of the participants in OMJ and GC groups were not statistically different $(p>0.05)$.

\section{Effects of artificial saliva on salivary flow rates}

The stimulated salivary flow rates of participants in both OMJ and GC groups showed an increasing trend after 1 and 2 months of interventions (Fig. 2). However, only the GC group showed statistically significant improvement at 2 months $(p=0.015)$. Nevertheless, there was no statistically significant difference in the salivary flow rates between groups at each time point.

\section{Effects of artificial saliva on saliva pH and buffering capacity}

As shown in Fig. 3a, participants with acidic saliva pH demonstrated a significant increase in saliva $\mathrm{pH}$ at 1 month after the interventions in both OMJ and GC groups ( $p=0.042$ and 0.027 , respectively). However, the saliva $\mathrm{pH}$ did not reach neutral value $(\mathrm{pH} 6.8)$ and remained acidic. There was no statistically significant difference in saliva $\mathrm{pH}$ between groups at each time point.

Likewise, the average saliva buffering capacity of participants with abnormal buffering capacity in the OMJ group showed an improvement from low (less than 4) to moderate level at 1 and 2 months as shown in Fig. 3b. The significant difference was detected at 1 month after the use of OMJ ( $p=$ $0.013)$. On the other hand, saliva buffering capacity of those in the GC group remained low without significant change.

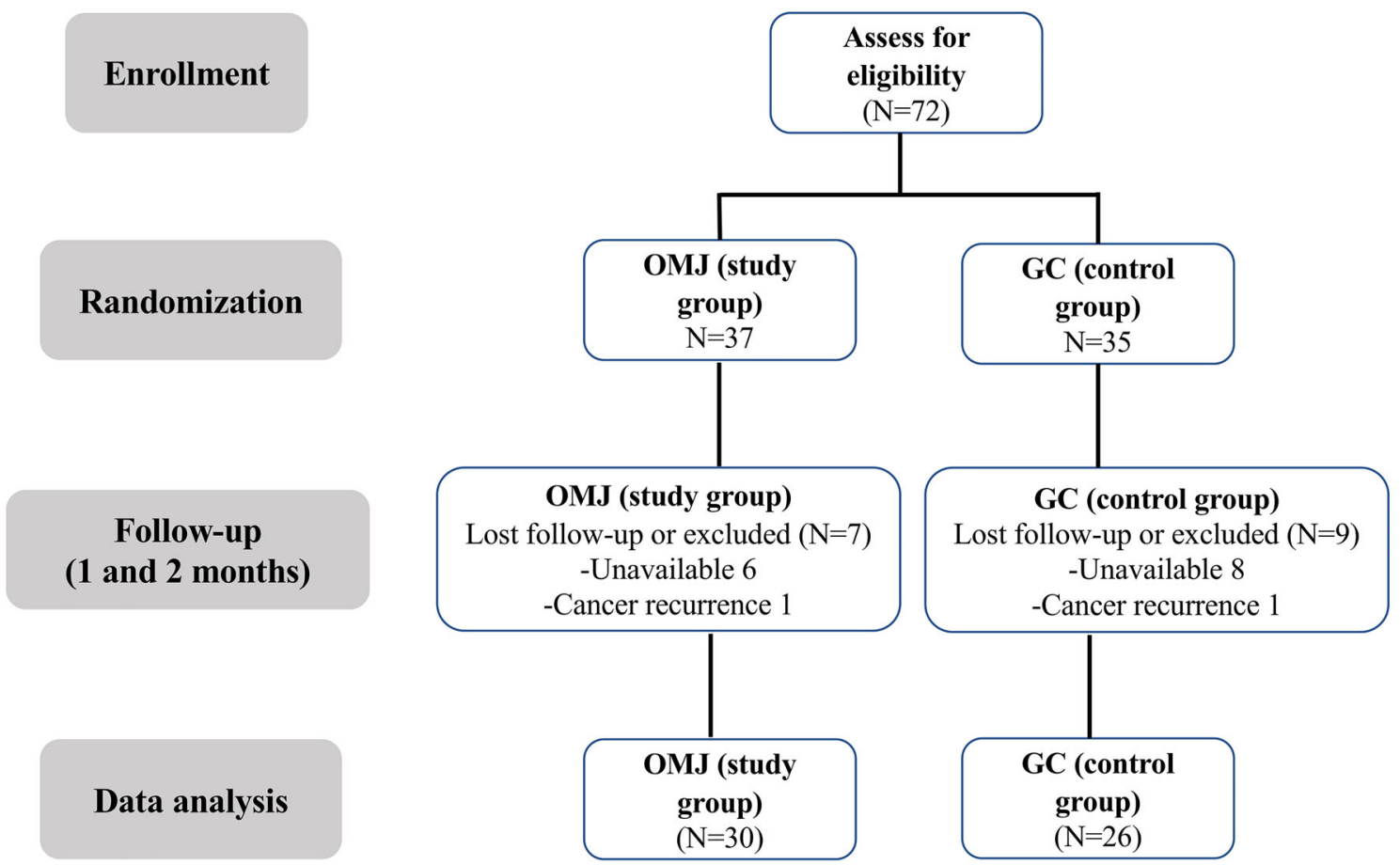

Fig. 1 Participants' flow chart of the randomized controlled study. Number of participants enrolled, dropped out, and included for data analysis are shown 
Table 1 Demographic characteristics and baseline data of the study population

\begin{tabular}{|c|c|c|c|}
\hline & $\mathrm{OMJ}(N=30)$ & $\mathrm{GC}(N=26)$ & $p$ value \\
\hline Sex, $N(\%)$ & & & $0.346^{\mathrm{C}}$ \\
\hline Male & $22(73.3)$ & $16(61.5)$ & \\
\hline Female & $8(26.7)$ & $10(38.5)$ & \\
\hline Age (years, average + SD) & $55.8 \pm 9.3$ & $56.9 \pm 10.7$ & $0.706^{\mathrm{T}}$ \\
\hline Cancer location, $N(\%)$ & & & $0.755^{\mathrm{F}}$ \\
\hline Nasopharynx & $10(33.3)$ & $10(38.5)$ & \\
\hline Oral cavity & $10(33.3)$ & $6(23.1)$ & \\
\hline Tongue & $5(16.7)$ & $5(19.2)$ & \\
\hline Larynx & $3(10)$ & $2(7.7)$ & \\
\hline Oropharynx & $2(6.7)$ & $1(3.9)$ & \\
\hline Others & 0 & $2(7.7)$ & \\
\hline Type of radiation, $N(\%)$ & & & $0.920^{\mathrm{C}}$ \\
\hline 2-dimensional & $20(66.7)$ & $17(65.4)$ & \\
\hline 3-dimensional & $10(33.3)$ & $9(34.6)$ & \\
\hline Duration after radiotherapy, $N(\%)$ & & & $0.791^{\mathrm{F}}$ \\
\hline Less than 6 months & $5(16.7)$ & $3(11.5)$ & \\
\hline 6 months to 1 year & $5(16.7)$ & $6(23.1)$ & \\
\hline 1 year and more & $20(66.7)$ & $17(65.4)$ & \\
\hline Denture use, $N(\%)$ & $6(20.0)$ & $6(23.1)$ & $0.780^{\mathrm{C}}$ \\
\hline Antifungal use within 1 year, $N(\%)$ & $2(6.7)$ & $4(15.4)$ & $0.401^{\mathrm{F}}$ \\
\hline Antibiotic use within 1 month, $N(\%)$ & $5(16.7)$ & $7(26.9)$ & $0.351^{\mathrm{C}}$ \\
\hline Subjective dry mouth score & $5.4 \pm 1.5$ & $5.1 \pm 1.5$ & $0.520^{\mathrm{T}}$ \\
\hline Salivary flow rates $(\mu \mathrm{l} / \mathrm{min})$ & $80.2 \pm 114.4$ & $49.1 \pm 66.5$ & $0.274^{\mathrm{M}}$ \\
\hline Saliva $\mathrm{pH}$ & $6.5 \pm 0.8$ & $6.3 \pm 0.9$ & $0.322^{\mathrm{T}}$ \\
\hline Saliva buffering capacity & $4.0 \pm 0.9$ & $4.1 \pm 1.1$ & $0.808^{\mathrm{T}}$ \\
\hline Candida carriers, $N(\%)$ & $27(90)$ & $21(80.8)$ & $0.451^{\mathrm{F}}$ \\
\hline
\end{tabular}

${ }^{\mathrm{F}}$ By Fisher's exact test

${ }^{\mathrm{C}}$ By Pearson chi-square test

${ }^{\mathrm{T}}$ By independent $t$ test

${ }^{\mathrm{M}}$ By Mann-Whitney $U$ test

${ }^{*}$ Statistically significant difference $(p<0.05)$
When analyzing changes in each subject, the OMJ group had significantly higher percentage of improved buffering capacity at 1 month than the GC group (Fig. 3c, $p=0.029$ ). Moreover, the proportion of participants with moderate buffering capacity was significantly increased in the OMJ group at 1 and 2 months (Fig. $3 \mathrm{~d}, p=0.013$ and 0.045 , respectively). On the other hand, participants in the GC group did not reveal significant changes in buffering capacity.

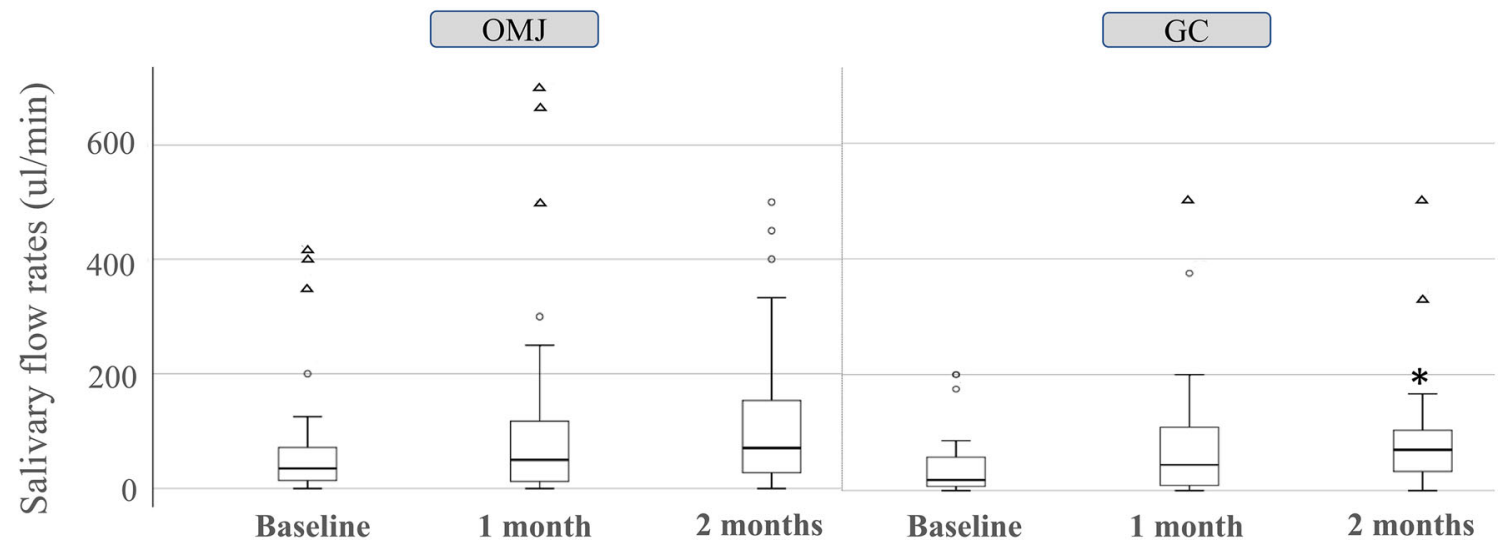

Fig. 2 Effect of artificial saliva on stimulated salivary flow rates. Box plot represents median and interquartile (IQ) range of salivary flow rates of participants in the OMJ and GC groups at baseline and after 1 and 2 months of interventions. Whiskers indicate the highest and lowest values no greater than 1.5 times the IQ range. Open circles and triangles

represent outliers (values between 1.5 and 3 times the IQ range) and extremes (values more than 3 times the IQ range), respectively. Asterisk (*) indicated a $p$ value of $<0.05$ by using related samples Friedman's test with Bonferroni correction for multiple comparisons. 

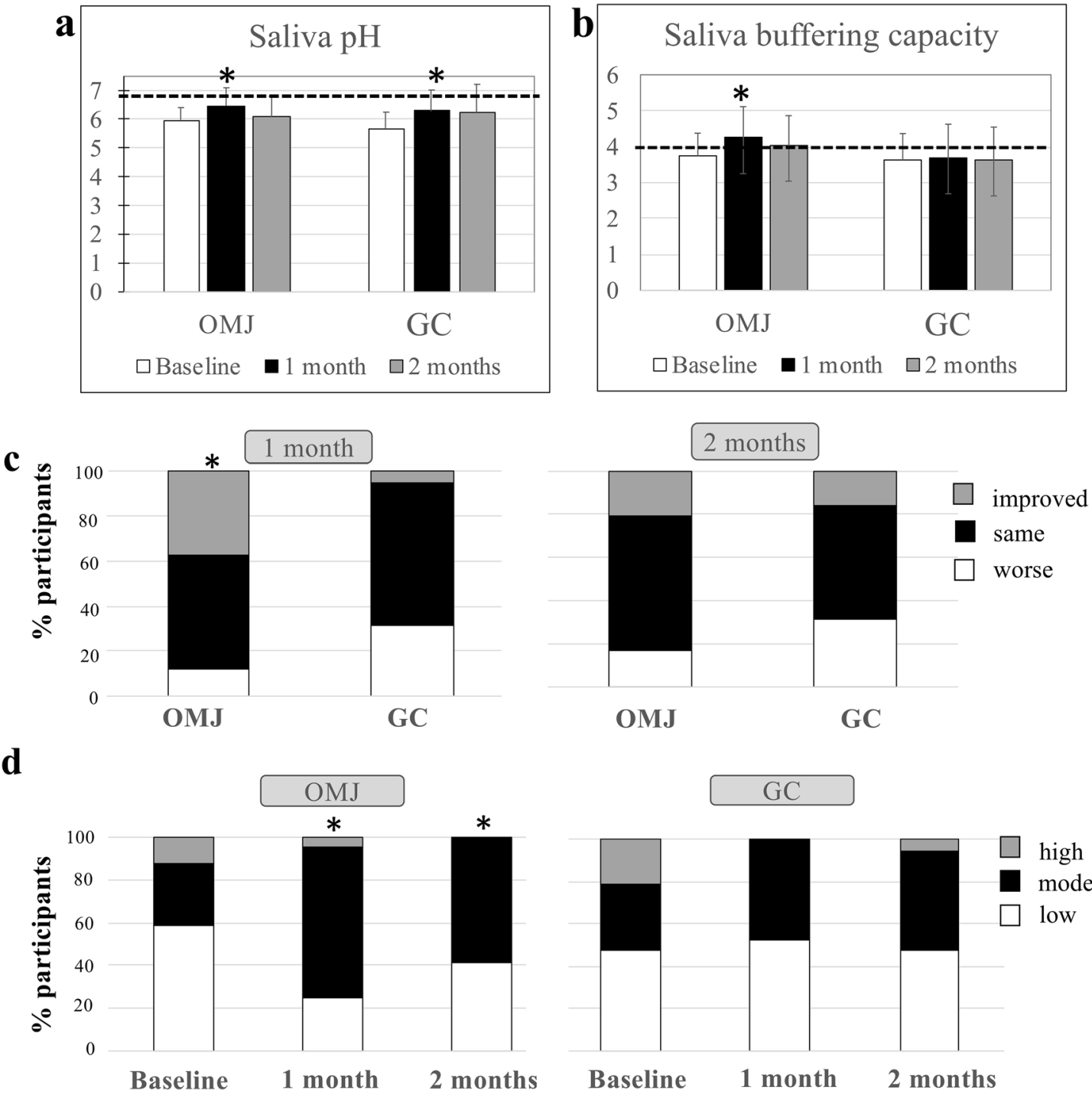

Fig. 3 Effect of artificial saliva on saliva $\mathrm{pH}$ (a) and buffering capacity (b-d). Average saliva $\mathrm{pH}$ (a) and buffering capacity ( $\mathrm{pH}$ after the addition of $\mathrm{HCl})(\mathbf{b})$ of participants in $\mathrm{OMJ}$ and $\mathrm{GC}$ groups at baseline and after 1 and 2 months of interventions. Asterisk $(*)$ indicates $p$ value $<0.05$ by using repeated measure ANOVA with Bonferroni correction for multiple comparisons. Dotted line represents the levels of neutral $\mathrm{pH}$ (a) and moderate buffering capacity (b). Comparison of changes in buffering

\section{Effects of artificial saliva on Candida colonization}

The prevalence of Candida carriage in the OMJ group decreased from 90 at baseline to $80 \%$ at 2 months, while those in GC slightly decreased from 80.8 to $76.9 \%$ at 2 months. Interestingly, the quantity of Candida $\operatorname{load}(\log \mathrm{CFU})$ among Candida carriers in both OMJ and GC groups slightly decreased at 1 and 2 months after using the products (Fig. 4a). However, no statistical significance was found. When we analyzed the changes in Candida quantity, the majority of participants in both groups showed decreased load at 1 and 2 months (70.4 and $74.1 \%$ for OMJ vs 57.1 and $61.9 \%$ for

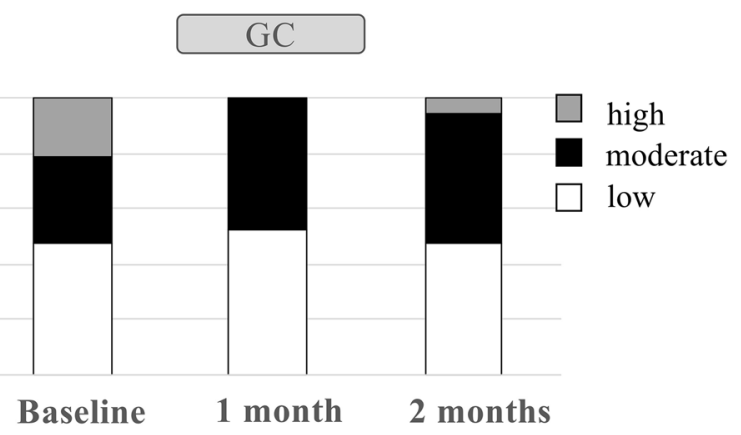

capacity between OMJ and GC groups after 1 and 2 months of interventions (c, d). Stacked bars (c) represent percentage of participants with the same, improved, or worse buffering capacity as compared with their own baseline. Stacked bars (d) represent percentage of participants with low, moderate, and high buffering capacity at each time point. Asterisk (*) indicates $p$ value $<0.05$ by using Fisher's exact test

GC at 1 and 2 months, respectively) as shown in Fig. 4b. The proportion of participants with decreased Candida quantity than baseline in the OMJ group appeared greater than that in the GC group; however, the difference was not statistically significant. Taken together, these data suggested that continuous use of artificial saliva might decrease the amount of Candida colonization.

\section{Effects of artificial saliva on Candida species}

Because multiple Candida species were reported to affect the complexity of infection and treatment, we also identified 
a

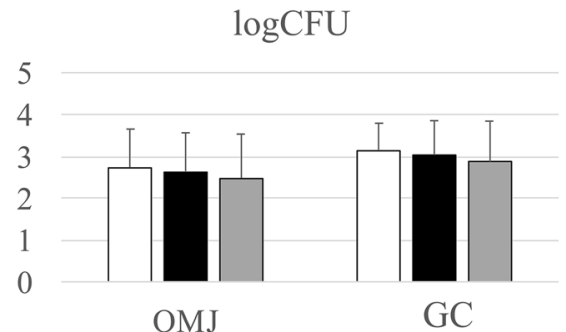

Baseline

1 month

2 months

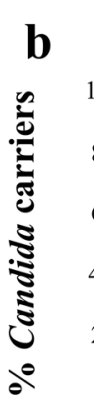

\section{2 months}

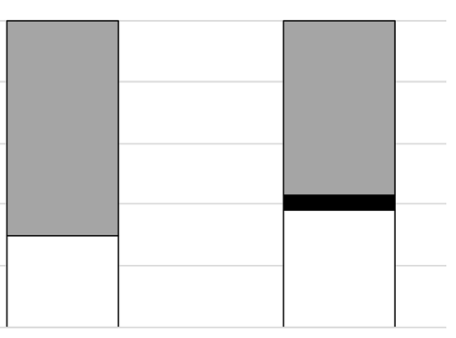

OMJ

GC
Fig. 4 Effect of artificial saliva on the quantity of Candida colonization in Candida carriers. Average $\log C F U$ (a) of Candida colonization in OMJ and GC groups at baseline and after 1 and 2 months of

Candida species colonized in the oral cavity of the participants who were Candida carriers. As shown in Fig. 5a, GC and OMJ significantly decreased the number of Candida

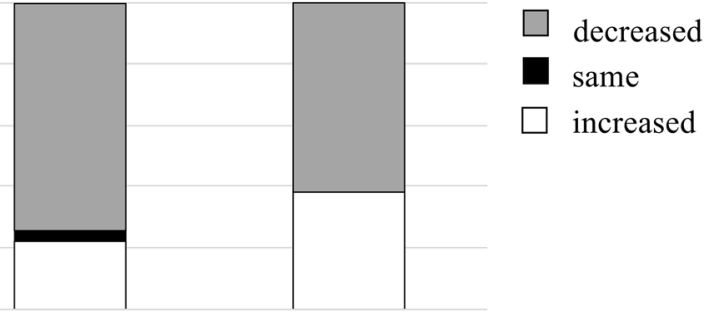

OMJ
GC

interventions. Stacked bars (b) represent percentage of Candida carriers with the same, increased, or decreased $\log \mathrm{CFU}$ as compared with their own baseline

species at 1 and 2 months $(p<0.0001$ and $p<0.01)$, respectively. Participants in the OMJ group had a reduced number of Candida species from 1-2 species to no detectable Candida

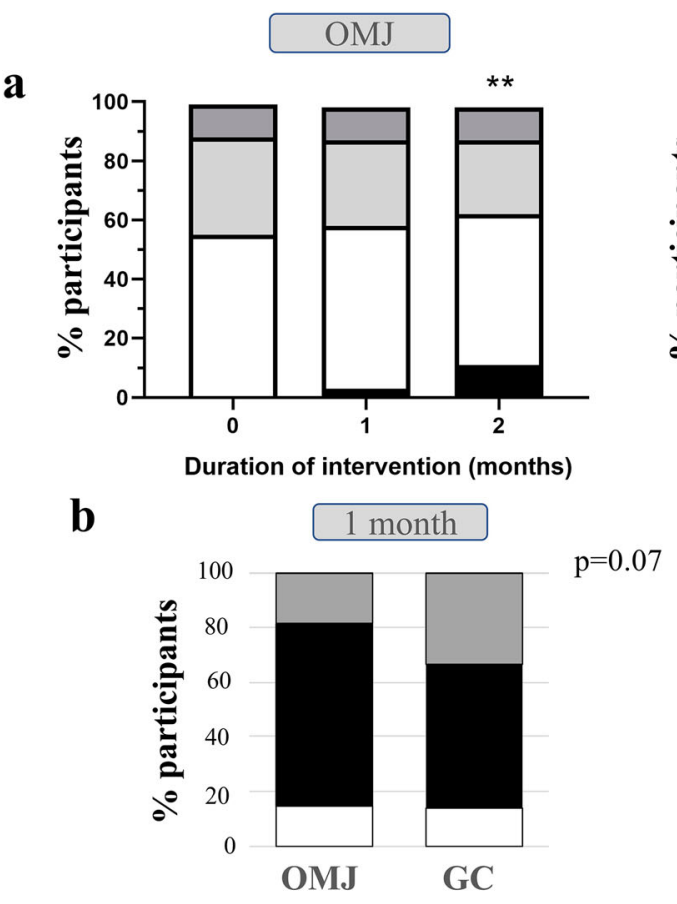

Fig. 5 Effects of artificial saliva on the number of Candida species in Candida carriers. Stacked bar represents a percentage of participants with no Candida colonization ( 0 species) or colonized with $1,2,3$, and 4 Candida species in OMJ and GC groups at 0,1 , and 2 months of interventions, and $\mathbf{b}$ percentage of participants with the increased, same,
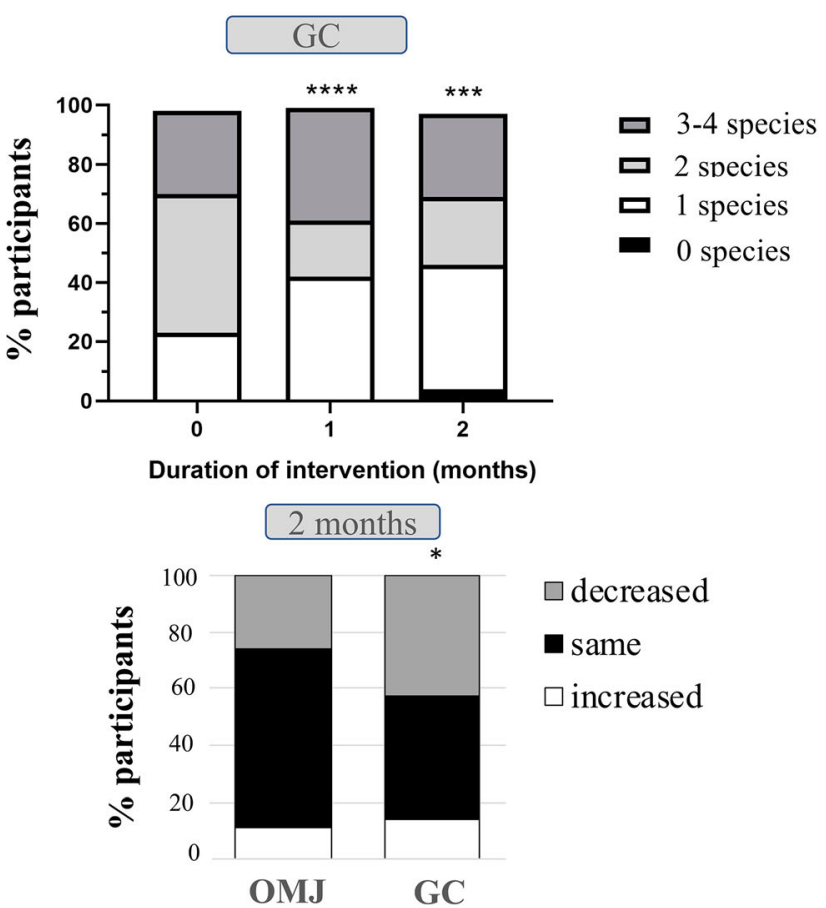

or decreased number of Candida species as compared with their own baseline in OMJ and GC groups at 1 month and 2 months of intervention. Asterisk, double asterisk, triple asterisk, and quadruple asterisk represent $p$ value $<0.05,0.01,0.001$, and 0.0001 , respectively, obtained from Chi-square test 
species, while the GC group had reduced mostly from 2 to 1 species.

In addition, when evaluating the changes in the number of Candida species in each individual, Fig. $5 \mathrm{~b}$ shows that the GC group had significantly higher percentage of participants with a decreased number of Candida species at 2 months after intervention, compared with that of the OMJ group $(p<0.05)$.

Figure 6 shows the prevalence of all Candida species detected in the participants and changes during the 2-month period of the trial. The most common Candida species colonized in the participants was $C$. albicans, followed by C. tropicalis, C. glabrata, and C. dubliniensis. After 2 months, the OMJ group demonstrated a slight decrease in the prevalence of C. albicans $(10 \%)$ and C. glabrata (10\%), while a small increase in C. dubliniensis $(6.67 \%)$ and no change in C. tropicalis were found. Participants in the GC group demonstrated a decrease in C. tropicalis (11.5\%) and C. glabrata (15.4\%) at 2 months, while no change in $C$. dubliniensis was observed. Interestingly, certain species, such as C. parapsilosis and Kodamaea ohmeri, were detected in both groups, but the presence may be transient.

\section{Correlation between saliva properties and Candida counts}

To investigate the relationship between saliva properties (salivary flow rates, $\mathrm{pH}$, and buffering capacity) and the quantity of Candida colonization ( $\log \mathrm{CFU}$ ) in Candida carriers, correlation analysis was performed (Fig. 7). At baseline, $\log \mathrm{CFU}$ showed significant negative correlation with saliva $\mathrm{pH}(p=$ 0.013), suggesting an increase in Candida colonization upon acidic environment. In addition, saliva $\mathrm{pH}$ had significant positive correlation with salivary flow rate at baseline and 1month follow-up $(p<0.01)$. Interestingly, after 2 -month interventions, significant negative correlation was found between Candida quantity $(\log \mathrm{CFU})$ and salivary flow rate $(p=$ 0.001 ), but not saliva $\mathrm{pH}$. No significant correlation was detected between Candida quantity and other saliva properties. The data suggested that the alteration of oral environment by the interventions could affect Candida colonization.

\section{Discussion}

Artificial saliva is commonly used to relieve dry mouth in various groups including post-radiotherapy head and neck cancer patients [17-19]. Though hyposalivation alters saliva properties leading to complications such as candidiasis $[1,30$, 31], most clinical studies of artificial saliva only focused on signs and symptoms of dry mouth [18, 20, 21, 23, 32, 33]. To our knowledge, the effect of artificial saliva on Candida species has never been documented. In this study, we reported for the first time that saliva gels (both OMJ and GC) could reduce the number of Candida species in xerostomic cancer patients. Since the higher the number of Candida species, the more difficult treatment can be $[7,15,16]$, the effect of saliva gels suggests a favorable outcome. Furthermore, previous reports on the effect of artificial saliva on saliva biochemical properties (flow rate, $\mathrm{pH}$, and buffering capacity) are inconclusive due to short-term duration and non-randomized design [34, 35]. In this study, we reported the results from a randomized controlled study with up to 2-month duration. Interestingly, both OMJ and GC improved salivary $\mathrm{pH}$ toward neutral one. Nevertheless, OMJ is superior to GC in its buffering capacity, while GC may better improve salivary flow rate. Interestingly, saliva $\mathrm{pH}$ and salivary flow rate were inversely correlated with Candida count. These findings suggested that modification of saliva properties may influence Candida colonization.

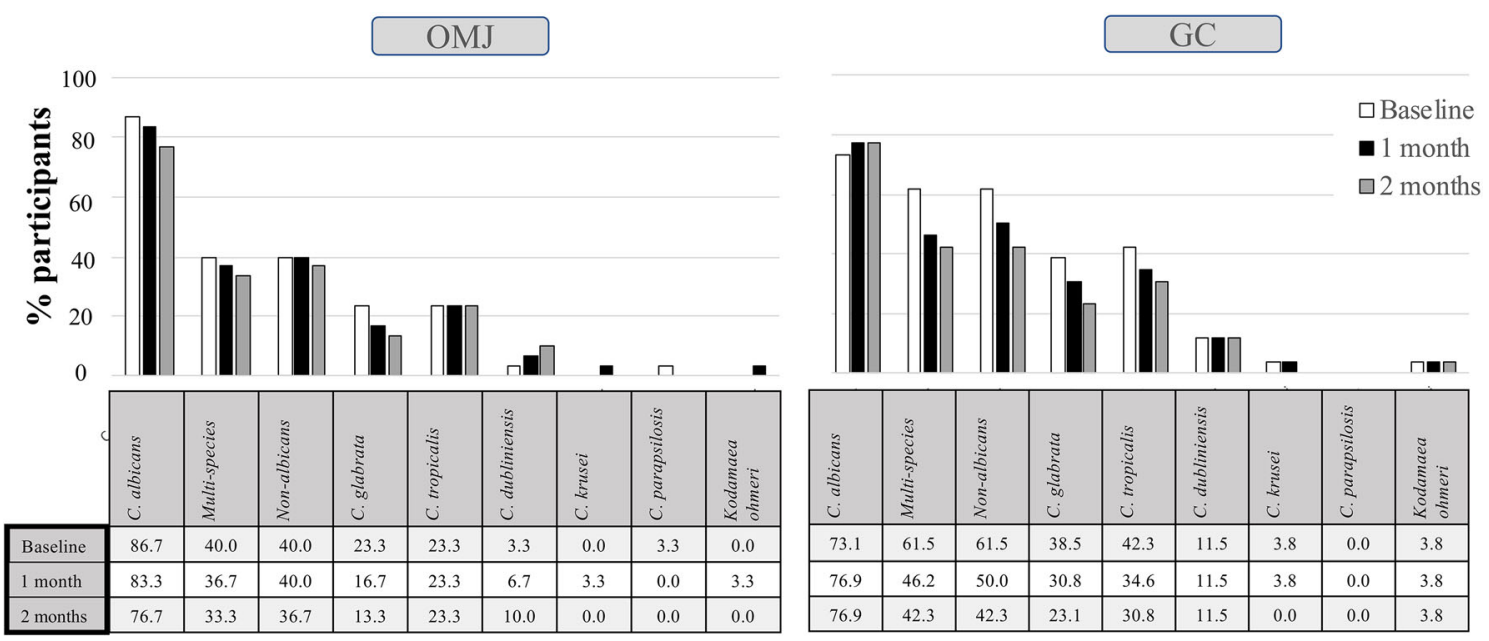

Fig. 6 Effects of artificial saliva on Candida species. The prevalence of various Candida species detected in OMJ and GC groups at baseline and after 1 and 2 months of interventions is shown 

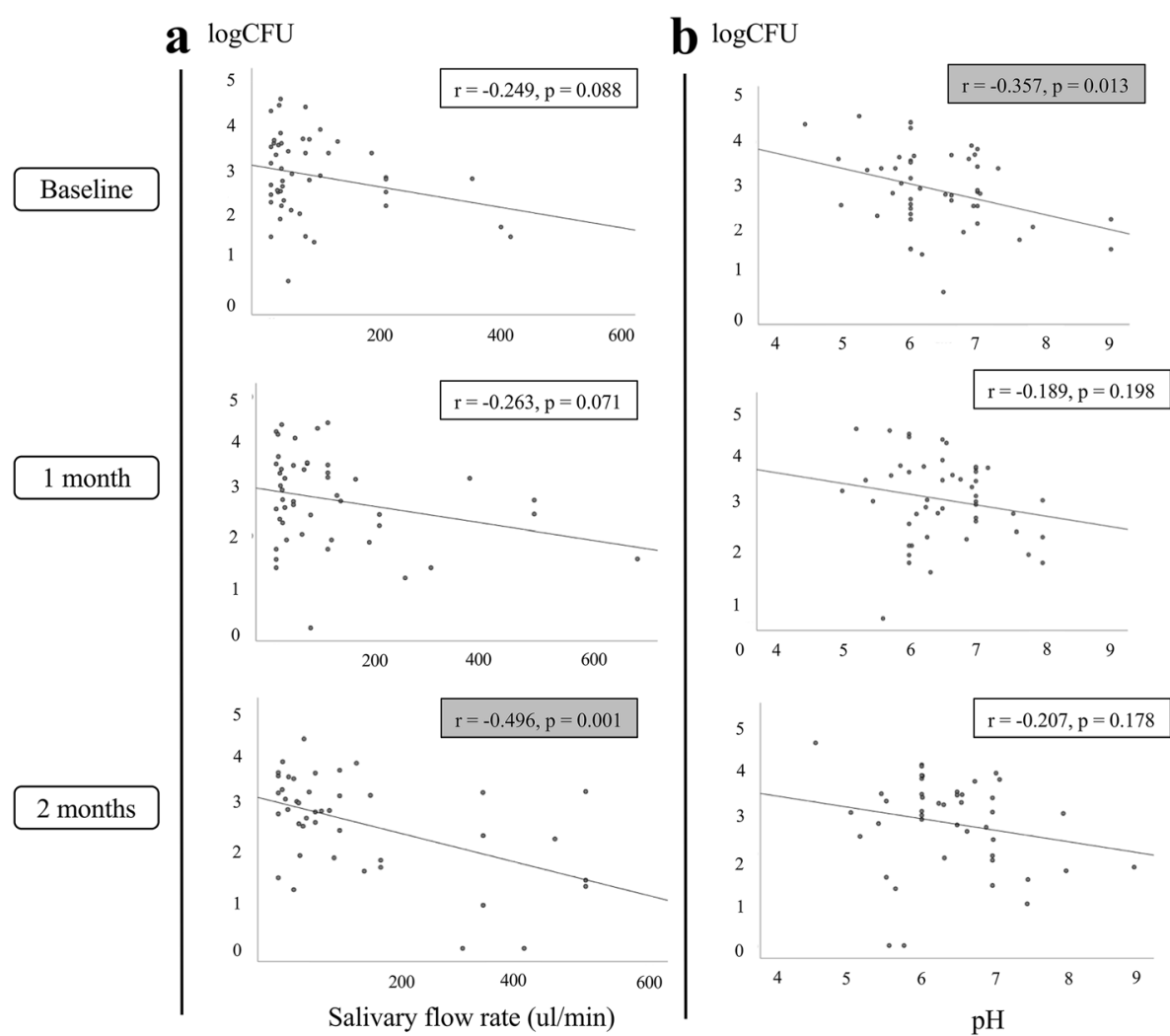

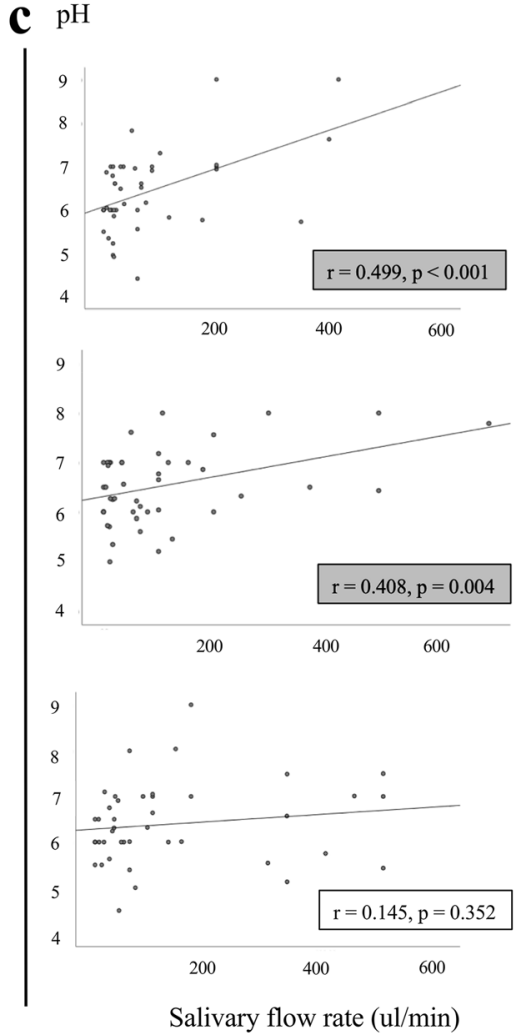

Salivary flow rate $(\mathrm{ul} / \mathrm{min})$
Fig. 7 Relationship of saliva properties and quantity of Candida colonization after artificial saliva use. Dot plot demonstrated correlation between Candida $\log$ CFU and salivary flow rate (a), Candida $\log C F U$ and saliva $\mathrm{pH}(\mathbf{b})$, and saliva $\mathrm{pH}$ and salivary flow rate (c) of Candida carriers at baseline and 1 and 2 months after intervention. $r$ and a $p$ value analyzed by Pearson correlation analysis shown in each plot. Grayshaded box indicated a $p$ value $<0.05$

to the oral mucosa and not recommended to be swallowed. On the other hand, OMJ could be held in the mouth and then swallowed to provide moisture both in the oral cavity and in the throat. Interestingly, the average saliva buffering capacity in OMJ users significantly improved at 1 month and shifted from low to moderate buffering capacity. In addition, a significantly higher proportion of participants in the OMJ group had improved buffering capacity than in the GC group. The average saliva buffering capacity of GC users remained low throughout the study. This could be because OMJ contains phosphate-buffering agent as its ingredient while GC gel does not. The improved buffering capacity was also observed in elderly patients after using OMJ [25]. In addition, a crossover study in participants with hyposalivation showed similar findings after using Biotene Dry Mouth Oral Rinse and a novel mouthwash product [36]. Because saliva buffering capacity plays an important role in maintaining a neutral oral environment, it would be of value to investigate the long-term effects of OMJ on oral and dental conditions in these patients.

The result of this study showed no significant difference between OMJ and the commercially available GC dry mouth gel in many aspects. First of all, salivary flow rates of participants in both groups appeared to improve after 1 and 2 months, although statistical significance was detected only reason why the gel is recommended for topical application 
in the GC group at 2 months. Similar finding was reported earlier in a study testing artificial saliva Optimoist in Sjogren's syndrome and post-radiotherapy head and neck cancer patients [34] as well as another study of $1 \%$ malic acid spray in xerostomic elderly patients [37]. However, another study reported no difference [38]. While the mechanism underlying this observation is unclear, we speculate that the increased lubrication and the ability to move the tongue and oral structures may stimulate saliva flow. In addition, flavors have been reported to promote saliva production [39]. Since GC dry mouth gel has higher viscosity and stronger flavor than OMJ, it may stimulate more saliva production. We also considered the functional recovery of salivary gland tissues as a possible mechanism of increased salivary flow rates. However, such recovery has been reported to occur mostly during 6 months to 1 year after radiation, and particularly with 3-dimensional radiotherapy $[2,40]$. Because the majority of our participants received 2-dimensional radiotherapy and had finished radiation for more than 1 year, the recovery during the study period would be unlikely.

Secondly, our results showed that saliva $\mathrm{pH}$ significantly increased in both OMJ and GC groups at 1 month, although not to a neutral value. This was in concordance with previous studies that evaluated the effects of Bioxtra gel in head and neck cancers and OMJ in elderly participants [25, 35]. In addition, Biotene Dry Mouth Oral Rinse and a novel mouthwash product were shown to improve saliva $\mathrm{pH}$ in xerostomic participants [36]. Both OMJ and GC products have neutral $\mathrm{pH}$, which may be important for the improved saliva $\mathrm{pH}$ observed in the participants. This finding may be clinically important because both salivary flow rates and saliva $\mathrm{pH}$ were shown to correlate with reduced Candida colonization [41-44].

Consistently with previous studies [7, 12, 45, 46], a high prevalence of Candida carriage was detected in postradiotherapy head and neck cancer patients in our study. We also found higher percentages of non-albicans species at baseline compared with healthy populations in other studies $[4,13$, $14,47]$. After the interventions, both OMJ and GC groups had a slight decrease in Candida $\log \mathrm{CFU}$ at 1 and 2 months. OMJ group appeared to have a higher percentage of users with decreased $\log \mathrm{CFU}$ than the GC group. However, the changes/differences did not reach statistical significance. The decrease in Candida colonization after the use of artificial saliva had been reported by a few studies [34, 35]. However, a study that tested the effects of Biotene products, which contain antimicrobial agents, lactoperoxidase and xylitol, did not show any improvement in Candida count in xerostomic postradiotherapy cancer patients [24]. This could be due to a short study time ( 2 weeks). The underlying mechanism of the reduction in Candida quantity has not been investigated. Both OMJ and GC dry mouth gel do not contain any antimicrobial agent. The possible explanation for reduced Candida counts in our study could be due to increased salivary flow rate and saliva $\mathrm{pH}$, and, therefore, better oral clearance [41, 42]. Correlation analysis seemed to support this hypothesis. At baseline, acidic saliva $\mathrm{pH}$ showed significant correlation with higher Candida quantity and low salivary flow rate. Both interventions led to increased salivary flow rate and saliva pH (Figs. 2 and 3a). At 2-month follow-up, Candida $\log$ CFU showed significant negative correlation with salivary flow rate, although no significant correlation was observed with saliva $\mathrm{pH}$ (Fig. 7). We did not find significant correlation between Candida $\log$ CFU and saliva buffering capacity, but the improvement in buffering capacity in the OMJ group may also help to neutralize the acidic saliva $\mathrm{pH}$. Because high amounts of Candida colonization were shown to be a risk factor of oral candidiasis [10], the apparent trend of decreasing Candida quantity after continuous use of artificial saliva suggests that it could decrease the risk of candidiasis. Further investigations with a larger number of participants and a longer follow-up time are warranted.

Similarly to previous reports, we also detected both C. albicans and non-albicans Candida species in postradiotherapy head and neck cancer patients [4, 7, 12, 48]. Continuous usage of both OMJ and GC dry mouth gel showed a decreasing trend in the number of Candida species and nonalbicans species colonized in the oral cavity, although the results were not statistically significant. This finding was noteworthy because colonization by non-albicans species and multiple Candida species was shown to be more resistant to treatment [7]. Specifically, it was reported that C. glabrata, C. tropicalis, and K. ohmeri can develop resistance to azole drugs $[15,16]$. Since our study observed a reduced number of Candida species after continuous use of either GC or OMJ, application of these saliva gels may prevent Candida colonization and improve treatment outcome. To our knowledge, our study is the first to report changes in the prevalence of Candida species after using artificial saliva. A longer-term study may be required in order to see a clear effect of artificial saliva on Candida colonization.

The strengths of this study were the parallel-group randomized controlled design and the long duration of 2 months, which provide a high level of evidence for clinical practice. To date, only a few randomized controlled trials have been conducted to investigate the effects of artificial saliva on oral health, and most studies used a crossover design and of shorter duration $[24,37,49]$. Moreover, the dry mouth characteristics of the participants were quite similar because we only included xerostomic patients due to definitive radiotherapy, which usually leads to severe hyposalivation. We matched the demographic and certain baseline characteristics of the participants between the two groups. The other characteristics that were not matched were also comparable. Although salivary flow rates of participants in the GC group appeared lower than those in OMJ groups at baseline, all participants were in severe hyposalivation state (flow rate of less than $700 \mu \mathrm{l} / \mathrm{min}$ ) 
[32], and no statistically significant difference between groups was detected.

This study carries certain limitations. First, a larger number of participants in the GC group were lost to follow-up, leaving unequal numbers of participants in both groups. Moreover, the saliva buffering capacity cannot be measured in some participants due to an insufficient amount of saliva (6 and 7 participants from OMJ and GC groups, respectively). Although we obtained adequate power for major outcome analyses, the sample size may not be enough to detect significant differences in certain variables such as Candida count. In addition, our 2month follow-up period may not be long enough to see clear effects on the quantity of Candida colonization, yet a trend of continued improvement was observed. Thus, a larger randomized controlled study with longer follow-up period is warranted.

To conclude, this study showed that OMJ and GC usage over a period of 2 months led to an improvement of saliva properties, a reduction in the number of Candida species, and a decreasing trend in Candida counts. OMJ achieves many properties of suitable artificial saliva, including lubrication, $\mathrm{pH}$ neutralization, and likely increase oral clearance. There were no statistically significant differences in these outcomes when OMJ was compared with the commercially available GC dry mouth gel. Further investigations on the long-term effects of OMJ on oral and dental health will be beneficial. The information is important for future development of artificial saliva and the management of xerostomic patients.

Acknowledgments The authors would like to thank all funding organizations and participants in the study. Also, we thank Ms. Sureeporn Muangsawat and Ms. Utamaporn Boonzong and all the staffs at Chonburi Cancer Hospital for their support.

Author contributions All authors contributed to the study conception and design. O. Chokchaitam and O. Mungkung were responsible for screening and recruitment of the participants. AL performed randomization. Data and clinical sample collection was performed by D. Trachootham, S. Tarapan, V. Sattabanasuk, S. Talungchit, W. Paemuang, and T. Phonyiam. S. Tarapan performed Candida species identification and statistical analyses under the supervision of A.-Lam-ubol and O. Matangkasombut. A. Lam-ubol wrote the manuscript, and all authors commented on previous versions of the manuscript. All authors read and approved the final manuscript.

Funding information This study was funded by the Dental Innovation Foundation under Royal Patronage, a non-profit organization. The foundation provided financial support and OMJ samples. In addition, Faculty of Dentistry, Srinakharinwirot University grant and Ratchadaphiseksomphot Endowment Fund (Research Unit on Oral Microbiology and Immunology) supported research funding. All experimental design, data collection, and data analysis were performed by the researchers independently without any involvement from the funding organization

\section{Compliance with ethical standards}

All procedures involving human participants in this study were in accordance with the ethical standards of the institutional research committee and with the 1964 Helsinki declaration and its later amendments or comparable ethical standards.

Informed consent was obtained from all individual participants included in the study.

Conflict of interest DT and AL have received a research grant from Dental Innovation Foundation under Royal Patronage. Other authors declare that they have no conflict of interest.

Open Access This article is licensed under a Creative Commons Attribution 4.0 International License, which permits use, sharing, adaptation, distribution and reproduction in any medium or format, as long as you give appropriate credit to the original author(s) and the source, provide a link to the Creative Commons licence, and indicate if changes were made. The images or other third party material in this article are included in the article's Creative Commons licence, unless indicated otherwise in a credit line to the material. If material is not included in the article's Creative Commons licence and your intended use is not permitted by statutory regulation or exceeds the permitted use, you will need to obtain permission directly from the copyright holder. To view a copy of this licence, visit http://creativecommons.org/licenses/by/4.0/.

\section{References}

1. Chambers MS, Garden AS, Kies MS, Martin JW (2004) Radiationinduced xerostomia in patients with head and neck cancer: pathogenesis, impact on quality of life, and management. Head Neck 26(9):796-807. https://doi.org/10.1002/hed.20045

2. Jensen SB, Vissink A, Limesand KH, Reyland ME (2019) Salivary gland hypofunction and xerostomia in head and neck radiation patients. J Natl Cancer Inst Monogr 2019(53). https://doi.org/10. 1093/jncimonographs/lgz016

3. Arrifin A, Heidari E, Burke M, Fenlon MR, Banerjee A (2018) The effect of radiotherapy for treatment of head and neck cancer on oral flora and saliva. Oral Health Prev Dent 16(5):425-429

4. Tarapan S, Matangkasombut O, Trachootham D, Sattabanasuk V, Talungchit S, Paemuang W, Phonyiam T, Chokchaitam O, Mungkung OO, Lam-Ubol A (2019) Oral Candida colonization in xerostomic postradiotherapy head and neck cancer patients. Oral Dis 25(7):1798-1808. https://doi.org/10.1111/odi.13151

5. Epstein JB, Thariat J, Bensadoun RJ, Barasch A, Murphy BA, Kolnick L, Popplewell L, Maghami E (2012) Oral complications of cancer and cancer therapy: from cancer treatment to survivorship. CA Cancer J Clin 62(6):400-422. https://doi.org/10.3322/ caac. 21157

6. Jham BC, da Silva Freire AR (2006) Oral complications of radiotherapy in the head and neck. Rev Bras Otorrinolaringol 72(5):704708

7. Karbach J, Walter C, Al-Nawas B (2012) Evaluation of saliva flow rates, Candida colonization and susceptibility of Candida strains after head and neck radiation. Clin Oral Investig 16(4):13051312. https://doi.org/10.1007/s00784-011-0612-1

8. Bensadoun RJ, Patton LL, Lalla RV, Epstein JB (2011) Oropharyngeal candidiasis in head and neck cancer patients treated with radiation: update 2011. Support Care Cancer 19(6):737-744. https://doi.org/10.1007/s00520-011-1154-4

9. Fanello S, Bouchara JP, Sauteron M, Delbos V, Parot E, MarotLeblond A, Moalic E, Le Flohicc AM, Brangerd B (2006) Predictive value of oral colonization by Candida yeasts for the onset of a nosocomial infection in elderly hospitalized patients. J Med Microbiol 55(Pt 2):223-228. https://doi.org/10.1099/jmm.0.461550 
10. Epstein JB, Pearsall NN, Truelove EL (1980) Quantitative relationships between Candida albicans in saliva and the clinical status of human subjects. J Clin Microbiol 12(3):475-476

11. Azizi A, Rezaei M (2009) Prevalence of Candida species in the oral cavity of patients undergoing head and neck radiotherapy. J Dent Res Dent Clin Dent Prospects 3(3):78-81. https://doi.org/10.5681/ joddd.2009.020

12. Thaweboon S, Thaweboon B, Srithavaj T, Choonharuangdej S (2008) Oral colonization of Candida species in patients receiving radiotherapy in the head and neck area. Quintessence Int 39(2):e52e57

13. Santiwongkarn P, Kachonboon S, Thanyasrisung P, Matangkasombut O (2012) Prevalence of oral Candida carriage in Thai adolescents. J Investig Clin Dent 3(1):51-55. https://doi. org/10.1111/j.2041-1626.2011.0089.x

14. Thanyasrisung P, Kesakomol P, Pipattanagovit P, YoungnakPiboonratanakit P, Pitiphat W, Matangkasombut O (2014) Oral Candida carriage and immune status in Thai human immunodeficiency virus-infected individuals. J Med Microbiol 63(Pt 5):753759

15. Sadeghi G, Ebrahimi-Rad M, Mousavi SF, Shams-Ghahfarokhi M, Razzaghi-Abyaneh M (2018) Emergence of non-Candida albicans species: epidemiology, phylogeny and fluconazole susceptibility profile. J Mycol Med 28(1):51-58. https://doi.org/10.1016/j. mycmed.2017.12.008

16. Papon N, Courdavault V, Clastre M, Bennett RJ (2013) Emerging and emerged pathogenic Candida species: beyond the Candida albicans paradigm. PLoS Pathog 9(9):e1003550. https://doi.org/ 10.1371/journal.ppat. 1003550

17. Lysik D, Niemirowicz-Laskowska K, Bucki R, Tokajuk G, Mystkowska J (2019) Artificial saliva: challenges and future perspectives for the treatment of xerostomia. Int J Mol Sci 20(13). https://doi.org/10.3390/ijms20133199

18. See L, Mohammadi M, Han PP, Mulligan R, Enciso R (2019) Efficacy of saliva substitutes and stimulants in the treatment of dry mouth. Spec Care Dentist 39(3):287-297. https://doi.org/10. $1111 / \mathrm{scd} .12370$

19. Dost F, Farah CS (2013) Stimulating the discussion on saliva substitutes: a clinical perspective. Aust Dent J 58(1):11-17. https://doi. org/10.1111/adj.12023

20. Oh DJ, Lee JY, Kim YK, Kho HS (2008) Effects of carboxymethylcellulose (CMC)-based artificial saliva in patients with xerostomia. Int J Oral Maxillofac Surg 37(11):1027-1031. https:// doi.org/10.1016/j.ijom.2008.06.006

21. Assery MKA (2019) Efficacy of artificial salivary substitutes in treatment of xerostomia: a systematic review. J Pharm Bioallied Sci 11(Suppl 1):S1-S12. https://doi.org/10.4103/jpbs.JPBS_220 18

22. Skrinjar I, Vucicevic Boras V, Bakale I, Andabak Rogulj A, Brailo V, Vidovic Juras D, Alajbeg I, Vrdoljak DV (2015) Comparison between three different saliva substitutes in patients with hyposalivation. Clin Oral Investig 19(3):753-757. https://doi.org/ 10.1007/s00784-015-1405-8

23. Shahdad SA, Taylor C, Barclay SC, Steen IN, Preshaw PM (2005) A double-blind, crossover study of Biotene Oralbalance and BioXtra systems as salivary substitutes in patients with postradiotherapy xerostomia. Eur J Cancer Care (Engl) 14(4):319326. https://doi.org/10.1111/j.1365-2354.2005.00587.x

24. Epstein JB, Emerton S, Le ND, Stevenson-Moore P (1999) A double-blind crossover trial of Oral Balance gel and Biotene toothpaste versus placebo in patients with xerostomia following radiation therapy. Oral Oncol 35(2):132-137. https://doi.org/10.1016/ s1368-8375(98)00109-2

25. Dalodom S, Lam-Ubol A, Jeanmaneechotechai S, Takamfoo L, Intachai W, Duangchada K, Hongsachum B, Kanjanatiwat P, Vacharotayangul P, Trachootham D (2016) Influence of oral moisturizing jelly as a saliva substitute for the relief of xerostomia in elderly patients with hypertension and diabetes mellitus. Geriatr Nurs 37(2):101-109. https://doi.org/10.1016/j.gerinurse.2015.10. 014

26. Nuchit S, Lam-Ubol A, Paemuang W, Talungchit S, Chokchaitam O, Mungkung OO, Pongcharoen T, Trachootham D (2020) Alleviation of dry mouth by saliva substitutes improved swallowing ability and clinical nutritional status of post-radiotherapy head and neck cancer patients: a randomized controlled trial. Support Care Cancer 28(6):2817-2828. https://doi.org/10.1007/s00520-01905132-1

27. Ericson D, Bratthall D (1989) Simplified method to estimate salivary buffer capacity. Scand J Dent Res 97:405-407

28. Mannarelli BM, Kurtzman CP (1998) Rapid identification of Candida albicans and other human pathogenic yeasts by using short oligonucleotides in a PCR. J Clin Microbiol 36(6):1634-1641

29. Mirhendi H, Makimura K, Khoramizadeh M, Yamaguchi H (2006) A one-enzyme PCR-RFLP assay for identification of six medically important Candida species. Nihon Ishinkin Gakkai Zasshi 47(3): 225-229

30. Braam PM, Roesink JM, Raaijmakers CP, Busschers WB, Terhaard CH (2007) Quality of life and salivary output in patients with head-and-neck cancer five years after radiotherapy. Radiat Oncol 2:3. https://doi.org/10.1186/1748-717X-2-3

31. Parliament MB, Scrimger RA, Anderson SG, Kurien EC, Thompson HK, Field GC, Hanson J (2004) Preservation of oral health-related quality of life and salivary flow rates after inverseplanned intensity- modulated radiotherapy (IMRT) for head-andneck cancer. Int J Radiat Oncol Biol Phys 58(3):663-673. https:// doi.org/10.1016/S0360-3016(03)01571-2

32. Epstein JB, Villines DC, Singh M, Papas A (2017) Management of dry mouth: assessment of oral symptoms after use of a polysaccharide-based oral rinse. Oral Surg Oral Med Oral Pathol Oral Radiol 123(1):76-83. https://doi.org/10.1016/j.oooo.2016.09. 008

33. Dirix P, Nuyts S, Vander Poorten V, Delaere P, Van den Bogaert W (2007) Efficacy of the BioXtra dry mouth care system in the treatment of radiotherapy-induced xerostomia. Support Care Cancer 15(12):1429-1436. https://doi.org/10.1007/s00520-006-0210-y

34. Rhodus NL, Bereuter J (2000) Clinical evaluation of a commercially available oral moisturizer in relieving signs and symptoms of xerostomia in postirradiation head and neck cancer patients and patients with Sjogren's syndrome. J Otolaryngol 29(1):28-34

35. Gookizadeh A, Emami H, Najafizadeh N, Roayaei M (2012) Clinical evaluation of BIOXTRA in relieving signs and symptoms of dry mouth after head and neck radiotherapy of cancer patients at Seyed-al-Shohada Hospital, Isfahan, Iran. Adv Biomed Res 1:72. https://doi.org/10.4103/2277-9175.102976

36. Takesh T, Ho J, Firmalino MV, Islip D, Anbarani A, Wilder-Smith P (2018) Effects of a novel formulation on oral biofilm, pH buffering, and gingival health in patients with dry mouth. Int J Dent 2018: 2748274-2748278. https://doi.org/10.1155/2018/2748274

37. Gomez-Moreno G, Cabrera-Ayala M, Aguilar-Salvatierra A, Guardia J, Ramirez-Fernandez MP, Gonzalez-Jaranay M, CalvoGuirado JL (2014) Evaluation of the efficacy of a topical sialogogue spray containing malic acid $1 \%$ in elderly people with xerostomia: a double-blind, randomized clinical trial. Gerodontology 31(4):274-280. https://doi.org/10.1111/ger.12034

38. Lapiedra RC, Gomez GE, Sanchez BP, Pereda AA, Turner MD (2015) The effect of a combination saliva substitute for the management of xerostomia and hyposalivation. J Maxillofac Oral Surg 14(3):653-658. https://doi.org/10.1007/s12663-015-0752-y

39. Karami Nogourani M, Janghorbani M, Kowsari Isfahan R, Hosseini Beheshti M (2012) Effects of chewing different flavored gums on salivary flow rate and pH. Int J Dent 2012:569327569324. https://doi.org/10.1155/2012/569327 
40. Nutting CM, Morden JP, Harrington KJ, Urbano TG, Bhide SA, Clark C, Miles EA, Miah AB, Newbold K, Tanay M, Adab F, Jefferies SJ, Scrase C, Yap BK, A'Hern RP, Sydenham MA, Emson M, Hall E, group Ptm (2011) Parotid-sparing intensity modulated versus conventional radiotherapy in head and neck cancer (PARSPORT): a phase 3 multicentre randomised controlled trial. Lancet Oncol 12(2):127-136. https://doi.org/10.1016/S14702045(10)70290-4

41. Torres SR, Peixoto CB, Caldas DM, Silva EB, Akiti T, Nucci M, Uzeda M (2002) Relationship between salivary flow rates and Candida counts in subjects with xerostomia. Oral Surg Oral Med Oral Pathol Oral Radiol Endod 93(2):149-154

42. Nadig SD, Ashwathappa DT, Manjunath M, Krishna S, Annaji AG, Shivaprakash PK (2017) A relationship between salivary flow rates and Candida counts in patients with xerostomia. J Oral Maxillofac Pathol 21(2):316

43. Al-Attas SA, Amro SO (2010) Candidal colonization, strain diversity, and antifungal susceptibility among adult diabetic patients. Ann Saudi Med 31(2):101-108

44. Balan P, Gogineni SB, N SK, Shetty V, Rangare AL, Castelino RL, K FA (2015) Candida carriage rate and growth characteristics of saliva in diabetes mellitus patients: a case-control study. J Dent Res Dent Clin Dent Prospects 9(4):274-279
45. Jain M, Shah R, Chandolia B, Mathur A, Chauhan Y, Chawda J, Mosby S, Bhagalia S (2016) The oral carriage of Candida in oral cancer patients of Indian origin undergoing radiotherapy and/or chemotherapy. J Clin Diagn Res 10(2):ZC17-ZC20

46. de Freitas EM, Nobre SAM, Pires MBO, Faria RVJ, Batista AUD, Bonan PRF (2013) Oral Candida species in head and neck cancer patients treated by radiotherapy. Auris Nasus Larynx 40(4):400 404

47. Pongsiriwet S, Iamaroon A, Sriburee P, Pattanaporn K, Krisanaprakornkit S (2004) Oral colonization of Candida species in perinatally HIV-infected children in northern Thailand. J Oral Sci 46(2):101-105

48. Singh GK, Capoor MR, Naid D, B KT (2017) Spectrum of fungal infection in head and neck cancer patients on chemoradiotherapy. J Egypt Natl Canc Inst 29(1):33-37

49. Barbe AG, Ludwar L, Hamacher S, Noack MJ (2019) Efficacy of a newly developed mouth gel for xerostomia relief-a randomized double-blind trial. Oral Dis 25(6):1519-1529. https://doi.org/10. 1111/odi.13105

Publisher's note Springer Nature remains neutral with regard to jurisdictional claims in published maps and institutional affiliations. 